ELECTRONIC RESEARCH ANNOUNCEMENTS OF THE AMERICAN MATHEMATICAL SOCIETY

Volume 3, Pages 93-98 (September 11, 1997)

S $1079-6762(97) 00030-9$

\title{
PREVALENCE OF NON-LIPSCHITZ ANOSOV FOLIATIONS
}

\author{
BORIS HASSELBLATT AND AMIE WILKINSON
}

(Communicated by Krystyna Kuperberg)

To the memory of Gunnar Hasselblatt, 19.8.1928-12.7.1997

\begin{abstract}
We give sharp regularity results for the invariant subbundles of hyperbolic dynamical systems and give open dense sets of codimension one systems where this regularity is not exceeded as well as open dense sets of symplectic, geodesic, and codimension one systems where the analogous regularity results of Pugh, Shub, and Wilkinson are optimal. We produce open sets of symplectic Anosov diffeomorphisms and flows with low transverse Hölder regularity of the invariant foliations almost everywhere. Prevalence of low regularity of conjugacies on large sets is a corollary. We also establish a new connection between the transverse regularity of foliations and their tangent subbundles.
\end{abstract}

\section{INTRODUCTION}

A diffeomorphism $f$ of a compact Riemannian manifold $M$ is called Anosov if the tangent bundle splits (necessarily uniquely and continuously) into two $D f$-invariant subbundles $T M=E^{u} \oplus E^{s}$, and there exist constants $C$ and $a<1$ such that

$$
\left\|D f^{n}(v)\right\| \leq C a^{n}\|v\| \text { and }\left\|D f^{-n}(u)\right\| \leq C a^{n}\|u\| \quad \text { for } v \in E^{s}, u \in E^{u}, n \in \mathbb{N} \text {. }
$$

Thus, for two nearby points positive or negative iterates move apart exponentially fast. Any hyperbolic matrix $A \in S L(m, \mathbb{Z})$ induces an Anosov diffeomorphism of the $m$-torus $\mathbb{T}^{m} ; E^{u}$ and $E^{s}$ are the expanding and contracting root spaces of $A$. The main subject of this paper is the regularity of the unstable and stable bundles $E^{u}$ and $E^{s}$, which bears in particular on the regularity of conjugacies.

Anosov systems are structurally stable, meaning that if $f: M \rightarrow M$ is Anosov and $g$ is $C^{1}$-close to $f$ then there is a unique homeomorphism $h: M \rightarrow M$ close to the identity such that $h \circ f=g \circ h$ (see, e.g., [KH, Corollary 18.2.4]). The conjugacy $h$ is rarely differentiable, for if $f^{n}(p)=p$ and $h$ is differentiable at $p$ then by the Chain Rule, $D h(p) D f^{n}(p)=D g^{n}(h(p)) D h(p)$. Thus $D f^{n}(p), D g^{n}(h(p))$ have the same eigenvalues, which is not a $C^{1}$-open condition.

In fact these eigenvalues also give an obstruction to $h$ being Lipschitz or having high Hölder exponent $\left[\varphi: X \rightarrow Y\right.$ is $C^{\alpha}$ at $x \in X$ or $\alpha$-Hölder (if $\alpha=1$ we say $\varphi$ is Lipschitz) if there exist $C, d>0$ such that

$$
d_{Y}\left(\varphi(x), \varphi\left(x^{\prime}\right)\right) \leq C d_{X}\left(x, x^{\prime}\right)^{\alpha} \text { whenever } d_{X}\left(x, x^{\prime}\right) \leq d ;
$$

Received by the editors May 9, 1997.

1991 Mathematics Subject Classification. Primary 58F15; Secondary 53C12.

Key words and phrases. Anosov system, hyperbolic system, invariant foliations, stable foliation, Anosov splitting, horospheric foliations, holonomy, Hölder structures, conjugacy. 
$\varphi$ is $C^{\alpha}$ on a set $S$ if it is $C^{\alpha}$ at every $x \in S$ ]: While conjugacies are uniformly bi- $C^{\beta}$ for some $\beta>0$ (see, e.g., [KH, Theorem 19.1.2]), i.e., for some $C, d>0$

$$
(1 / C) d_{M}\left(x, x^{\prime}\right)^{1 / \beta} \leq d_{M}\left(h(x), h\left(x^{\prime}\right)\right) \leq C d_{M}\left(x, x^{\prime}\right)^{\beta} \text { for } d_{M}\left(x, x^{\prime}\right) \leq d,
$$

differing eigenvalue data will affect the Hölder exponent. As an illustration of this relationship, consider the related (non-Anosov) example of the expanding map $f(x)=\lambda x$ on $\mathbb{R}$. Suppose that $g:(-\epsilon, \epsilon) \rightarrow \mathbb{R}, g(0)=0,0<\lambda<\mu:=g^{\prime}(0)$ and $h \circ f=g \circ h$, where $h$ is bi- $C^{\alpha}$. Then

$$
h\left(f^{n}\left(x_{0}\right)\right)=g^{n}\left(h\left(x_{0}\right)\right) \approx \mu^{n} h\left(x_{0}\right)=\left(\lambda^{n} x_{0}\right)^{\alpha}\left(h\left(x_{0}\right) / x_{0}^{\alpha}\right)=C\left(f^{n}\left(x_{0}\right)\right)^{\alpha}
$$

for $\alpha:=\log \mu / \log \lambda<1$, i.e., $h$ is not Lipschitz.

A similar argument gives an obstruction to Lipschitz conjugacy between Anosov diffeomorphisms, but it is not a very sensitive one; if $g$ is $C^{1}$-close to $f$ then the periodic data are not very different and thus do not preclude a relatively high Hölder exponent. Furthermore these data are no obstruction to higher regularity off the countable set of periodic points (in fact, in dimension two they are the only obstructions to smooth conjugacy $[\mathrm{LM}]$ ). It was brought to our attention by John Franks that low regularity of the Anosov splitting (Theorem 3) provides a different and effective mechanism for showing that the Hölder exponent of many conjugacies arbitrarily close to the identity may be very low on a very large set:

Theorem 1. For $\alpha \in(0,1)$ there is a linear symplectic Anosov diffeomorphism $A$ and $a C^{1}$-neighborhood $U$ of $A$ of symplectic diffeomorphisms such that for a $C^{1}$-open $C^{\infty}$-dense set of $f \in U$ the conjugacy to $A$ is almost nowhere bi-C ${ }^{\alpha}$.

In his proof of ergodicity for volume-preserving Anosov diffeomorphisms, Anosov showed that there is always an $\alpha>0$ such that $E^{u}, E^{s}$ are uniformly $C^{\alpha}$; i.e., if

$$
H_{C, d}^{\alpha}:=\left\{p \in M \mid d_{B}\left(E^{u}(p), E^{u}(q)\right) \leq C d_{M}(p, q)^{\alpha} \text { if } d_{M}(p, q) \leq d\right\},
$$

for a smooth metric $d_{B}$ on subbundles, then $M=H_{C, d}^{\alpha}$ for some $C, d$. He did not estimate $\alpha$ [A2]. Hirsch-Pugh-Shub [HPS] estimated $\alpha$ via global contraction and expansion rates of $D f$, and Hasselblatt [H1] obtained better estimates from local data and showed that they are not exceeded for a generic symplectic Anosov system, or the geodesic flow of a generic negatively curved metric. We give optimal regularity results for subbundles and foliations and show that they are quite sharp and extreme nonsmoothness is quite common in the manifold and the space of Anosov systems. We

- sharpen the regularity results in [H1], [H3], [H4] and extend the sharpness statement to a wider class of Anosov systems (Theorem 4),

- show generic sharpness of the holonomy estimates in [PSW] (Theorems 5,4),

- find large sets of Anosov systems on which the regularity of $E^{u}, E^{s}, W^{u}$ and $W^{s}$ is low on a large set of points (Theorems 3 and 2, Proposition 7 ).

Our construction is elementary; it relies on a simple locally defined obstruction. Say that $S \subset M$ is negligible if $M \backslash S$ is residual and $\mu(S)=0$ for any ergodic invariant probability measure $\mu$ that is fully supported, i.e., positive on open sets.

Theorem 2. For $\alpha \in(0,1)$ there is a $C^{1}$-open set of symplectic Anosov flows and diffeomorphisms for which $E^{u}$ and $E^{s}$ are $C^{\alpha}$ only on a negligible set.

Many homotopy classes of Anosov systems contain such open sets. Note that if $\varphi$ is continuous and $S$ is dense, then $\varphi$ is $C^{\alpha}$ on $S$ if (1) holds for all $x, x^{\prime} \in S$, so these results show that no restrictions to large sets are very regular. 
An Anosov flow $\varphi^{t}: M \rightarrow M$ is a fixed point free flow with $D \varphi^{t}$-invariant splitting $T M=E^{s u} \oplus E^{s s} \oplus\langle\dot{\varphi}\rangle$, where $D \varphi^{t}$ expands and contracts $E^{s u}$ and $E^{s s}$, respectively. Examples are suspensions (mapping torus) of Anosov diffeomorphisms (cf. [KH, Section 0.3]) and geodesic flows (on the unit tangent bundle) of compact Riemannian manifolds with negative sectional curvature $[\mathrm{A} 1, \S 22]$ or $[\mathrm{KH}$, Theorem 17.6.2], where the foliations are called horospheric foliations. The weak stable and unstable bundles $E^{\ell}:=E^{s \ell} \oplus\langle\dot{\varphi}\rangle$ are tangent to weak foliations $W^{\ell}, \ell=s, u$.

The regularity of $E^{s}$ and $E^{u}$ is closely related to that of the foliations $W^{u}$, $W^{s}$ tangent to $E^{u}, E^{s}$ [A1, Theorem 8], [F], which have smooth leaves but low transverse regularity. Regularity is measured by examining the natural locally defined homeomorphism between two sufficiently close smooth disks transverse to the foliation (determined by following the leaves and called the holonomy map). If, for any two transversals from a compact family, the holonomy map is Hölder with exponent $\alpha$ and a uniformly bounded constant, then the foliation is said to be $C^{\alpha}$. Pugh-Shub-Wilkinson [PSW] showed that $W^{u}$ and $W^{s}$ are $C^{\alpha}$, with the same $\alpha$ as found for the subbundles in [H1]. There the exponent was not shown to be optimal.

Theorem 3. For $\alpha \in(0,1)$ there is a $C^{1}$-open set of symplectic Anosov flows and diffeomorphisms whose unstable and stable holonomies are almost nowhere $C^{\alpha}$.

By [H1] there generically is a periodic point in $M$ where $\alpha$ cannot exceed the predicted value. This left open the possibility that a larger $\alpha$ might work on the rest of the manifold. Anosov [A1, p. 201] has an example of an Anosov diffeomorphism where $E^{u}$ is almost nowhere $C^{2 / 3+\epsilon}$, for any $\epsilon>0$. ( $\left(E^{u}\right.$ is almost nowhere $C^{\alpha}$ with respect to $\mu$ if $\mu\left(\bigcup_{C, d} H_{C, d}^{\alpha}\right)=0$; see (2). $)$ In our examples regularity is also low on a large set, but stably so.

An optimal regularity estimate is interesting for several reasons. The regularity of the foliations is related to that of the conjugacy to a linear system, hence Theorem 1. Also, $E^{u}$ and $E^{s}$ are invariants of a smooth system that show a marked lack of smoothness measured by the largest possible exponent $\alpha$-if they are $C^{1}$ one can take $\alpha=1$ (conversely, when $\alpha=1$ the subbundles are often $C^{1}$ ). It can be related to dimension characteristics (see, e.g., [SS]). Work on stable ergodicity in partially hyperbolic systems [GPS], [W], [PS] used invariant subbundles with $\alpha$ close to 1. The regularity of horospheric foliations bears directly on that of Busemann functions [G]; smoothness characterizes locally symmetric metrics [BCG].

If $f^{n}(p)=p$ let $\mu_{f}(p)<\mu_{s}(p)<1<\nu_{s}(p)<\nu_{f}(p)$ be the minimal and maximal absolute values of the eigenvalues of $D_{p} f^{n}$ in and outside the unit circle and

$$
B_{\text {per }}^{u}(f):=\inf \left\{\frac{\log \mu_{s}(p)-\log \nu_{s}(p)}{\log \mu_{f}(p)} \mid f^{n}(p)=p \text { for some } n \in \mathbb{N}\right\} .
$$

E.g., if $f$ is symplectic then $\nu_{s} \mu_{s}=1$, so $B_{\text {per }}^{u}(f)=2 \inf _{p} \log \mu_{s}(p) / \log \mu_{f}(p)$ is close to 2 if and only if the contraction rates $\mu_{s}$ and $\mu_{f}$ are close together. $f$ is said to be transitive if it has a dense orbit; this holds for Anosov systems preserving volume (by ergodicity) and for systems with $\operatorname{codim}\left(E^{s}\right)=1[\mathrm{~N}]$. Denote by $C^{r}$ the space of $C^{\lfloor r\rfloor}$ maps whose $\lfloor r\rfloor$ th derivatives have modulus of continuity $O\left(x^{r-\lfloor r\rfloor}\right)$.

Theorem 4. 1. If $f$ is transitive Anosov and $B_{p e r}^{u}(f) \notin \mathbb{N}$ then $E^{u} \in C^{B_{p e r}^{u}(f)}$.

2. If $f$ is transitive Anosov and $B_{p e r}^{u}(f) \in \mathbb{N}$ then $E^{u} \in C^{B_{p e r}^{u}(f)-1, O(x|\log x|)}$.

3. 1 and 2 hold for flows.

4. For an open dense set of symplectic diffeomorphisms and flows the regularity of $E^{u}$ and $W^{u}$ is at most that asserted in 1 and 2. 
5. For an open dense set of diffeomorphisms and flows with $\operatorname{codim}\left(E^{s}\right)=1$ the regularity of $E^{u}$ and $W^{u}$ is at most that asserted in 1 and 2.

6. 1-3 hold for hyperbolic sets.

7. Of the metrics on a compact manifold with sectional curvature $\leq-k^{2}$ and injectivity radius $\geq \log 2 / k$ an open dense set has horospheric foliations (hence structure at infinity) of at most the regularity claimed in 1 and 2.

The results about the subbundles are due to the first author; the others then follow from Theorem 5. Theorem 4 encompasses some known results. For example, an area-preserving diffeomorphism or geodesic flow in dimension 2 has $C^{1, O(x|\log x|)}$ foliations [HK]. Transitivity can be replaced by bunching along all orbits [H4].

A foliation tangent to a Hölder subbundle may not have Hölder holonomy maps, even when the leaves are uniformly smooth [W]. This is related to the fact that a Hölder vector field need not be uniquely integrable; trajectories near nonunique ones can move apart rapidly. Surprisingly, the converse holds; Hölder regularity of holonomy maps implies (essentially) the same regularity for the tangent subbundles:

Theorem 5. If the holonomies of a foliation $\mathcal{F}$ of a Riemannian manifold $M$ with uniformly $C^{n+1}\left(C^{\infty}\right)$ leaves are $C^{\alpha-}:=\bigcap_{\beta<\alpha} C^{\beta}$ then $T \mathcal{F}$ is $C^{\alpha n /(n+1)-}\left(C^{\alpha-}\right)$.

Suppose $\lambda$ is a Borel probability measure on $M$ whose $\mathcal{F}$-conditionals are absolutely continuous and such that for some family $\left\{D_{x}\right\}_{x \in M}$ of smooth transversals and for $\lambda$-almost every $x, y$ with $y \in \mathcal{F}(x)$, the $\mathcal{F}$-holonomy map $h: D_{x} \rightarrow D_{y}$, $h(p)=\mathcal{F}(p) \cap D_{y}$ is absolutely continuous and $C^{\alpha-}$ a.e. (with respect to Riemannian volume on $\left.D_{x}\right)$. Then $T \mathcal{F}$ is $C^{\alpha n /(n+1)-}\left(C^{\alpha-}\right) \lambda$-a.e.

This is proved by induction on derivatives along leaves. There might be a true difference in regularity between holonomy and tangent distribution when the leaves are not $C^{\infty}$ but this question remains open. Note that Theorem 3 now follows from Theorem 2 because the foliations are absolutely continuous: If the unstable subbundle is almost nowhere $C^{\alpha-\epsilon}$ the invariant set where the holonomies are $C^{\alpha}$ cannot have full measure by Theorem 5 , hence is a null set by ergodicity.

Our techniques need failure of bunching everywhere, not just at a periodic point:

Definition 6. $f$ is called $\alpha$-u-spread if there are $E^{f s} \subseteq E^{m s} \subsetneq E^{s}$ and constants $\mu_{f}, \mu_{s}, \nu$, with $\nu \mu_{f}^{\alpha}<\mu_{s}$, such that $\left\|D f_{\Gamma_{E^{f s}}^{n}}\right\|<\operatorname{cst} . \mu_{f}^{n},\left\|D f^{-n}(v)\right\|<\operatorname{cst} . \mu_{s}^{-n}\|v\|$ for $v \in E^{s} \backslash E^{m s}$, and $\left\|D f_{\uparrow_{E^{u}(x)}^{-n}}\right\|>\operatorname{cst} . \nu^{-n}$ for all $x$.

This means the Mather spectrum has annuli in $\left\{|z|<\mu_{f}\right\}$ and $\left\{\mu_{s}<|z|<1\right\}$ and an annulus overlapping $\{1<|z|<\nu\}$, and is an open condition $[\mathrm{P}]$. For example, $A:=\left(\begin{array}{cc}B & 0 \\ 0 & B^{\lfloor 2 / \alpha\rfloor+1}\end{array}\right)$, where $B=\left(\begin{array}{ll}2 & 1 \\ 1 & 1\end{array}\right)$, induces a symplectic Anosov automorphism of $\mathbb{T}^{4}$. Both $A$ and $A^{-1}$ are $\alpha$-u-spread. We obtain Theorem 2 from

Proposition 7. In a small neighborhood of an $\alpha$-u-spread symplectic or codimension one Anosov diffeomorphism or flow the systems whose unstable subbundle is $C^{\alpha}$ only on a negligible set are $C^{1}$-open $C^{\infty}$-dense.

If a metric of negative curvature on a compact manifold has $\alpha$-u-spread geodesic flow, then in a small neighborhood of this metric there is a $C^{3}$-open $C^{\infty}$-dense set of metrics whose horospheric subbundles are $C^{\alpha}$ on a negligible set only.

This gives Theorem 2 for $E^{u}$ or $E^{s}$ and the intersection of open sets is open. Suspending these examples proves the result for flows. No $\alpha$-u-spread geodesic flow 
is known to us. Perturbations of volume-preserving (e.g., linear) codimension one flows and diffeomorphisms have $C^{1}$ subbundles [A1, p. 11], hence are not examples.

To define the obstruction assume $\operatorname{codim}\left(E^{s}\right)=1$ for simplicity and at $x \in M$ choose coordinates which split as $\mathbb{R} \times \mathbb{R}^{s}$, with $\mathbb{R} \times\{0\}$ corresponding to $W^{u}(x)$ and $\{0\} \times \mathbb{R}^{s}$ to $W^{s}(x)$. Accordingly, the differential of $f$ at $y \in W^{s}(x)$ is

$$
D_{y} f=\left(\begin{array}{cc}
a & 0 \\
B & C
\end{array}\right)
$$

because $W^{s}(x)$ is preserved by $f$. Represent $E^{u}(y)$ as the graph of a linear map $D: \mathbb{R} \rightarrow \mathbb{R}^{s}$ or the image of $\left(\begin{array}{l}1 \\ D\end{array}\right): \mathbb{R} \rightarrow \mathbb{R} \times \mathbb{R}^{s}$. Then $D_{y} f\left(E^{u}\right)$ is the image of

$$
D_{y} f\left(\begin{array}{l}
1 \\
D
\end{array}\right)=\left(\begin{array}{ll}
a & 0 \\
B & C
\end{array}\right)\left(\begin{array}{l}
1 \\
D
\end{array}\right)=\left(\begin{array}{c}
a \\
B+C D
\end{array}\right)=\left(\begin{array}{c}
1 \\
(B+C D) / a
\end{array}\right) a,
$$

which is unchanged when we reparametrize the preimage by $a^{-1}$, so by invariance $E^{u}(f(y))$ is the graph of $f^{*} D:=(B+C D) / a$. If $z_{i}:=f^{i}(y)$ then $D\left(z_{1}\right)=\left(B\left(z_{0}\right)+\right.$ $\left.C\left(z_{0}\right) D\left(z_{0}\right)\right) / a\left(z_{0}\right)$ or $D\left(z_{i+1}\right)=\left(B\left(z_{i}\right)+C\left(z_{i}\right) D\left(z_{i}\right)\right) / a\left(z_{i}\right)$. If $f$ is $\alpha$-u-spread then for $y \in W_{\text {loc }}^{f s}(x)$ (the local fast stable leaf of $x$ defined by the adapted coordinate neighborhood) $C$ is lower block triangular. Denote the upper left $k \times k$ block corresponding to the complement of $E^{m s}$ by $c . B$ and $D$ are column vectors whose top $k$ entries define column vectors $b$ and $d$, and $d\left(z_{i+1}\right)=\left(b\left(z_{i}\right)+c\left(z_{i}\right) d\left(z_{i}\right)\right) / a\left(z_{i}\right)$. If $\xi_{z_{0}}^{n}:=\prod_{i=0}^{n-1} c\left(z_{n-i-1}\right), \eta_{z_{0}}^{n}:=\prod_{i=0}^{n-1} a\left(z_{i}\right)^{-1}$, and $\Delta_{z_{0}}^{n}:=-\sum_{i=0}^{n-1}\left(\xi_{z_{0}}^{i+1}\right)^{-1} b\left(z_{i}\right)\left(\eta_{z_{0}}^{i}\right)^{-1}$ then $\left|\left(\eta_{z_{0}}^{i}\right)^{-1}\right| \leq \operatorname{cst} . \nu^{i},\left\|\left(\xi_{z_{0}}^{i}\right)^{-1}\right\| \leq \operatorname{cst} . \mu_{s}^{-i},\left\|b\left(z_{i}\right)\right\| \leq \operatorname{cst} .\left\|z_{i}\right\| \leq \operatorname{cst} . \mu_{f}^{i}$, and $\mu_{f} \nu \leq \nu \mu_{f}^{\alpha}<\mu_{s}$, so the $\Delta_{z_{0}}^{n}$ converge uniformly, and the obstruction

$$
O(x):=\sup \left\{\left\|d(z)-\Delta_{z}^{f}\right\| \mid z \in W_{\text {loc }}^{f s}(x)\right\}
$$

is continuous in $x$ and $f$.

For an $\alpha$-u-spread periodic point $x$ take $y \in W_{\text {loc }}^{f s}(x)$ with a neighborhood $U$ such that $U \cap\left\{f^{i}(y) \mid i \in \mathbb{Z}\right\}=\{y\}$ and $x \notin U[\mathrm{H}$, Proposition 4.1]. Let $J$ be a perturbation of the identity on $M$ supported in $U \backslash\{y\}$ such that $D_{y} J=I+\epsilon e$ in adapted coordinates at $x$, where the only nonzero entry of $e$ is $e_{21}=1$. Then $J \circ f$ has $x$ periodic and $y \in W_{\text {loc }}^{f s}(x)$ not returning to $U$ with unstable subspace $J\left(\begin{array}{l}1 \\ D\end{array}\right)=\left(\begin{array}{l}1 \\ D\end{array}\right)+(0, \epsilon, 0, \ldots, 0)^{t}$, so $d(y) \neq \Delta_{y}^{f}=\Delta_{y}^{J \circ f}$ and $O \neq 0$ (stably).

\section{REFERENCES}

[A1] D. V. Anosov, Geodesic flows on closed Riemann manifolds with negative curvature, Proc. Steklov Inst. 90 (1967). MR 36:7157, MR 39:3527

[A2] D. V. Anosov, Tangential fields of transversal foliations in "U-systems", Mat. Zametki 2 (1967), no. 5, 818-823. MR 39:3523

[BCG] Gérard Besson, Gilles Courtois, Sylvestre Gallot, Minimal entropy and Mostow's rigidity theorems, Ergodic Theory and Dynamical Systems 16 (1996), no. 4, 623-649. MR 97e:58177

[F] Neil Fenichel, Asymptotic stability with rate conditions, Indiana University Math. Journal 23 (1974), 1109-1137; 26 (1977), no. 1, 81-93. MR 49:4036, MR 54:14002

[GPS] Matthew Grayson, Charles Pugh, Michael Shub, Stably ergodic diffeomorphisms, Annals of Mathematics (2) 140 (1994), no. 2, 295-329. MR 95g:58128

[G] Leon W. Green, The generalized geodesic flow, Duke Mathematical Journal 41 (1974), 115-126. MR 51:6885 
[H1] Boris Hasselblatt, Regularity of the Anosov splitting and of horospheric foliations, Ergodic Theory and Dynamical Systems, 14 (1994), no. 4, 645-666. MR 95j:58130

[H2] Boris Hasselblatt, Horospheric foliations and relative pinching, Journal of Differential Geometry 39 (1994), no. 1, 57-63. MR 95c:58137

[H3] Boris Hasselblatt, Periodic bunching and invariant foliations, Mathematical Research Letters 1 (1994), no. 5, 597-600. MR 95h:58097

[H4] Boris Hasselblatt, Regularity of the Anosov splitting II, Ergodic Theory and Dynamical Systems, 17 (1997), 169-172. CMP 97:10

[HPS] Morris Hirsch, Charles Pugh, Michael Shub, Invariant manifolds, Lecture Notes in Mathematics 583, Springer-Verlag, 1977. MR 58:18595

[HK] Steven Hurder, Anatole Katok, Differentiability, rigidity, and Godbillon-Vey classes for Anosov flows, Publications IHES 72 (1990), 5-61. MR 92b:58179

[KH] Anatole Katok, Boris Hasselblatt, Introduction to the modern theory of dynamical systems, Cambridge University Press, 1995. MR 96c:58055

[LM] Rafael de la Llave, Roberto Moriyon, Invariants for smooth conjugacy of hyperbolic dynamical systems. IV, Communications in Mathematical Physics 116 (1988), no. 2, 185192. MR 90h:58064

[N] Sheldon Newhouse, On codimension one Anosov diffeomorphisms, American Journal of Mathematics 92 (1970), 761-770. MR 43:2741

[P] Yakov Pesin, On the existence of invariant fiberings for a diffeomorphism of a smooth manifold, Math. USSR Sbornik 20 (1973), no. 2, 213-222. MR 49:8049

[PS] Charles Pugh, Michael Shub, Stably ergodic dynamical systems and partial hyperbolicity, Journal of Complexity, to appear

[PSW] Charles Pugh, Michael Shub, Amie Wilkinson, Hölder foliations, Duke Mathematical Journal, 86 (1997), no. 3, 517-546. CMP 97:07

[SS] Jörg Schmeling, Rainer Siegmund-Schulze, Hölder continuity of the holonomy maps for hyperbolic basic sets, I, Ergodic theory and related topics, III (Güstrow, 1990), pp. 174191, Springer lecture notes in mathematics 1514, Springer, Berlin, 1992. MR 93j:58104

[W] Amie Wilkinson, Stable ergodicity of the time-one map of a geodesic flow, Ergodic Theory and Dynamical Systems, to appear

Department of Mathematics, Tufts University, Medford, MA 02155-5597

E-mail address: bhasselb@tufts.edu

Department of Mathematics, Northwestern University, Evanston, IL 60208-2730

E-mail address: wilkinso@math.nwu.edu 